\title{
Espectrofluorimetria comparada à cromatografia líquida de alta eficiência na detecção de ocratoxina A em café
}

\section{Ochratoxin A detection by spectrofluorimetry compared with high performance liquid c hromatography in coffee}

\author{
Ricardo Marcelo Reche Ribeiro ${ }^{1}$; Simone Fujii'; ${ }^{2}$ Marcelo Silva ${ }^{3}$; \\ Andréia Botega Nogari ${ }^{4}$; Maria Brígida dos Santos Scholz; \\ Elisabete Yurie Sataque Ono ${ }^{6}$; Osamu Kawamura ${ }^{7}$; Elisa Yoko Hirooka ${ }^{8}$
}

\section{Resumo}

\begin{abstract}
A cafeicultura brasileira apresenta grande importância ao mercado interno e comércio exterior. A qualidade e sanidade do café dependem da contaminação fúngica, com ênfase as espécies produtoras de ocratoxina A (OTA), uma nefrotoxina de efeito deletério à saúde pública. A necessidade de contínuo monitoramento e controle de OTA em produtos agrícolas requer metodologia analítica simples, rápida e acessível. O trabalho teve como objetivo padronizar um método espectrofluorimétrico para detecção de OTA visando monitoramento em grãos de café, correlacionando-o com a cromatografia líquida de alta eficiência (CLAE). A aplicabilidade da espectrofluorimetria foi avaliada em café artificialmente contaminado com OTA (10, 20 e $50 \mathrm{ng} / \mathrm{g}$ ), comparando os resultados obtidos com a CLAE. Embora a fluorimetria tenha apresentado resultados insatisfatórios, com recuperação de 40,14 \% e coeficiente de correlação de 0,79 em relação a CLAE, i.e. baixa confiabilidade e eficiência inadequada, a técnica constitui perspectiva para prosseguir o estudo inserindo novos ensaios referentes à sua padronização e certificação.
\end{abstract}

Palavras-chave: Ocratoxina A, café, CLAE, espectrofluorimetria

\begin{abstract}
The Brazilian coffee production plays important role both in the national and oversea trade. The quality and sanity of coffee depend on fungal contamination, with emphasis on ochratoxin A (OTA) producing species, which is a nephrotoxin with hazardous effect in public health. The exigency on continuous monitoring and control of OTA in agricultural commodity requires a simple, rapid and accessible analytical methodology. The objective of this research was to set up the spectrofluorimetric method for OTA detection intended for monitoring in coffee, which was developed in data-correlation with high efficiency liquid chromatography (HPLC). The performance of spectrofluorimetric analysis was evaluated using artificially contaminated coffee (10,20 and $50 \mathrm{ng} / \mathrm{g})$, and the data compared with HPLC. Although of the fault of former technique, with recovery of $40.14 \%$ and correlation coefficient of 0.79 when compared to HPLC, i.e. low reliability and inadequate efficiency, the data showed perspective to follow the improvement inserting new tool, and further standardization and certification.
\end{abstract}

Key words: Ochratoxin A, coffee, HPLC, spectrofluorimetry

\footnotetext{
Mestre em Ciência de Alimentos, Depto. Ciência e Tecnologia de Alimentos, Centro de Ciências Agrárias, Universidade Estadual de Londrina-UEL, Londrina-PR. Email: recheribeiro@yahoo.com.br.

2 Doutora em Ciência de Alimentos, Depto. Ciência e Tecnologia de Alimentos, CCA, UEL, Londrina-PR.

3 Mestre em Biotecnologia, Depto. Bioquímica e Biotecnologia, CCE, UEL, Londrina-PR.

4 Graduada em Farmácia, UEL, Londrina-PR.

5 Instituto Agronômico do Paraná-IAPAR, Londrina-PR.

6 Docente do Depto. Bioquímica e Biotecnologia, UEL, Londrina-PR.

7 Docente de Food Hygienology, Faculty of Agriculture, KAGAWA University, Kida-gun, Kagawa-JP.

8 Docente do Depto. Ciência e Tecnologia de Alimentos, UEL, Londrina-PR.

* Autor para correspondência
} 


\section{Introdução}

A cafeicultura brasileira destaca-se no mercado interno e externo, representando importante parcela das exportações (TOLEDO, 2000). A qualidade de café está intimamente relacionada com a fitossanidade do fruto, repercutindo diretamente na economia do país e saúde do consumidor (PETRACCO, 1998).

O café está exposto à infestação natural por uma variedade de microrganismos, com ênfase a fungos produtores de ocratoxina A - OTA (FINKGREMMELS; JAHN; BLOM, 1995; JODBAUER; MAIER; LINDNER, 2002). A OTA constitui um metabólito secundário produzido por Aspergillus spp. e Penicillium spp., cuja relevância se justifica pelo envolvimento em intoxicações animais e na etiologia de doenças renais em humano (FINK-GREMMELS; JAHN; BLOM, 1995; JODBAUER; MAIER; LINDNER, 2002). As propriedades toxicológicas de OTA incluem nefrotoxicidade, teratogenicidade, citotoxicidade e genotoxicidade (GROSSO et al., 2003).

O limite máximo de OTA recomendado pela União Européia varia entre 5 a $20 \mathrm{ng} / \mathrm{g}$ para café verde, 3 a $10 \mathrm{ng} / \mathrm{g}$ para café torrado e 4 a $10 \mathrm{ng} / \mathrm{g}$ para café solúvel (FOOD AND AGRICULTURE ORGANIZATION OF UNITED NATIONS, 2006). Considerando o baixo limite permitido para OTA em alimentos, seu monitoramento requer $\mathrm{o}$ desenvolvimento de método analítico com alta especificidade e sensibilidade, sendo rapidez, reprodutibilidade, exatidão e precisão essenciais na diagnose de micotoxicologia humana e animal (CHU, 1984; ESKOLA; KOKKONEN; RIZZO, 2002; JODBAUER; MAIER; LINDNER, 2002).

O método oficial descrito em "Association of Official Analytical Chemists” (AOAC) para análise quantitativa de micotoxinas é a Cromatografia Líquida de Alta Eficiência (CLAE). No entanto, a demanda considerável de reagentes de alto grau de pureza, equipamento/manutenção de alto custo, tempo de análise significativo e supervisão por técnicos especializados, restringe o emprego na rotina laboratorial (SYDENHAM; SHEPHARD, 1996; FURLANI; OLIVEIRA; SOARES, 1999). A espectrofluorimetria destaca-se por oferecer simplicidade, sensibilidade, baixo custo, uso reduzido de solventes tóxicos e a ausência de contato do aparelho com estes solventes constituindo uma alternativa promissora para análise de micotoxinas em alimentos.

O trabalho teve por objetivo desenvolver um método alternativo de espectrofluorimetria para análise de OTA em café, visando conferir aos laboratórios a capacidade de monitorar maior número de amostras em sistema simplificado acoplada ao fluxo contínuo.

\section{Material e Métodos}

Preparação de amostras

Duzentos gramas de grãos de café (Coffea arabica, cultivar Sachimor Amarelo) foram previamente secos a $50^{\circ} \mathrm{C}$ por 16 horas até atingir $12 \%$ de umidade, triturados para 48 mesh em moinho laboratorial (A10, Janke \& Kunkel IKA ${ }^{\circledR}$ Labortechnik, Germany), e armazenados a " $20^{\circ} \mathrm{C}$.

\section{Extração de OTA}

A extração de OTA procedeu-se conforme descrito por Pittet e Royer (2002). Cinqüenta gramas de café triturados foram adicionadas de ácido fosfórico 0,1 M (25 mL) e diclorometano (125 mL), seguido de agitação (150 rpm - $60 \mathrm{~min}$ ) e filtração (Whatman $\mathrm{n}^{\circ} 1$ ). A seguir $20 \mathrm{~mL}$ do filtrado foi adicionado de bicarbonato de sódio a $3 \%(20 \mathrm{~mL})$ e 1-octanol (3 gotas). Após agitação, a solução aquosa $(18 \mathrm{~mL})$ foi acidificada ( $\mathrm{pH} 2,0)$ com ácido fosfórico $85 \%$, seguido de adição de clorofórmio $(5 \mathrm{~mL})$ e agitação (30 seg). A camada clorofórmica foi coletada e adicionada de $1,0 \mathrm{~g}$ de sulfato de sódio anidro $\left(\mathrm{Na}_{2} \mathrm{SO}_{4}\right)$. Volume de $3,5 \mathrm{~mL}$ da fase orgânica foi seca a $40{ }^{\circ} \mathrm{C}$ sob fluxo de $\mathrm{N}_{2}$ e ressuspendido em $100 \mu \mathrm{L}$ de clorofórmio. 


\section{Limpeza de extrato (CCD)}

Volume de $60 \mu \mathrm{L}$ de resíduo preparado conforme item acima foi aplicado como ponto em placa cromatográfica $(20$ × $20 \mathrm{~cm}$, Sílica gel 60 - MERCK) e desenvolvida com fase móvel constituída de tolueno:acetato de etila:ácido fórmico (50:40:10, v/v/ v). A placa foi visualizada sob luz UV (366 nm) e, a banda correspondente a OTA raspada, introduzida em tubo e adicionada de $2 \mathrm{~mL}$ de metanol. A mistura foi sonicada por $1 \mathrm{~min}$, filtrada (MILLIPORE -HV, $0,45 \mu \mathrm{m})$, seca a $40{ }^{\circ} \mathrm{C}$ sob fluxo de $\mathrm{N}_{2}$ e destinada à análise por CLAE e espectrofluorimetria.

\section{Cromatografia líquida de alta eficiência (CLAE)}

As amostras foram analisadas conforme descrito por Pittet et al. (1996). O resíduo foi ressuspendido em $200 \mu \mathrm{L}$ de acetonitrila:água $(1: 1, \mathrm{v} / \mathrm{v})$ e, $20 \mu \mathrm{L}$ injetado no sistema de CLAE (isocrático de fase reversa), consistindo de bomba (Shimadzu LC10$\mathrm{AD}$ ), detector de fluorescência (Shimadzu RF535) com comprimento de onda de excitação e emissão de 335 e $475 \mathrm{~nm}$, respectivamente, e coluna Nucleosil $100 \mathrm{C}_{18}(250 \mathrm{~mm}$ x $4 \mathrm{~mm}$, Merck, Darmstadt, Alemanha). A fase móvel consistiu de acetonitrila:água:ácido acético (99:99:2, v/v/v) sob fluxo de $0,8 \mathrm{~mL} / \mathrm{min}$. O limite de detecção para OTA $(0,80 \mathrm{ng} / \mathrm{g})$ foi calculado como 3 vezes o desvio padrão do branco da amostra obtido de sete repetições (SOARES, 2006). A concentração de OTA foi determinada utilizando curva padrão confeccionada na faixa de 5,0 ng/mL a $100,0 \mathrm{ng} / \mathrm{mL}$ de OTA.

\section{Espectrofluorimetria}

$\mathrm{O}$ resíduo foi ressuspendido s em $300 \mu \mathrm{L}$ de acetonitrila:água $(1: 1, \mathrm{v} / \mathrm{v})$ e, $200 \mu \mathrm{L}$ transferido para microplaca de poliestireno (96 poços) para análise por espectrofluorimetria (VARIAN-FLR), sob comprimento de onda de excitação e emissão de 335 e $475 \mathrm{~nm}$, respectivamente. A concentração de OTA foi determinada utilizando curva padrão confeccionada na faixa de 5,0 a $50,0 \mathrm{ng} / \mathrm{mL}$ de OTA. A aplicabilidade da técnica foi avaliada, comparando com a CLAE.

\section{Estudo de recuperação de OTA}

Grãos de café negativos para OTA $(<0,80$ ng/g, determinada por CLAE) foram artificialmente contaminados com OTA (500 ng/mL em metanol, Sigma Chemical Co., St Louis, MO, USA) para as concentrações de 10, 20 e $50 \mathrm{ng} / \mathrm{g}$. As amostras foram extraídas após repouso de $24 \mathrm{~h}$ a $25^{\circ} \mathrm{C}$ e, analisadas por espectrofluorimetria e CLAE. O estudo de recuperação consistiu de duas repetições em duplicata.

\section{Resultados e Discussão}

A Figura 1 mostra a curva padrão de OTA obtida por CLAE e espectrofluorimetria. Na CLAE, a curva padrão foi confeccionada utilizando seis concentrações de OTA $(5,10,25,50,75$ e 100 ng/ $\mathrm{mL})$ em acetonitrila:água $(1: 1, \mathrm{v} / \mathrm{v})$, obtendo-se equação da reta $\mathrm{y}=1798,4 \mathrm{x}-1619,6$ com coeficiente linear $\left(r^{2}\right)$ de 0,9984 e limite de detecção de 0,8 ng/g (Figura 1A). A técnica mostrou-se sensível e adequada para análise de OTA perante os níveis máximos permitidos, considerando que FOOD AND AGRICULTURE ORGANIZATION OF UNITED NATIONS (2006) adota limite de 5 a $20 \mathrm{ng} / \mathrm{g}$ para café verde, 3 a $10 \mathrm{ng} / \mathrm{g}$ para café torrado e 4 a 10 $\mathrm{ng} / \mathrm{g}$ para café solúvel. Na espectrofluorimetria utilizou-se seis concentrações de OTA $(5,10,20,30$, 40 e $50 \mathrm{ng} / \mathrm{mL})$ em acetonitrila:água $(1: 1, \mathrm{v} / \mathrm{v})$, obtendo-se equação da reta $\mathrm{y}=0,2729 \mathrm{x}-1,2153$, coeficiente linear de 0,8094 (Figura 1B). A baixa relação de linearidade obtida (80,94 \%) apontou que $19,06 \%$ de variabilidade na potência de fluorescência (eixo y) não foi explicada pela variabilidade da concentração de OTA (eixo x), indicando provável interferência de outros fatores que afetaram a potência de fluorescência. 

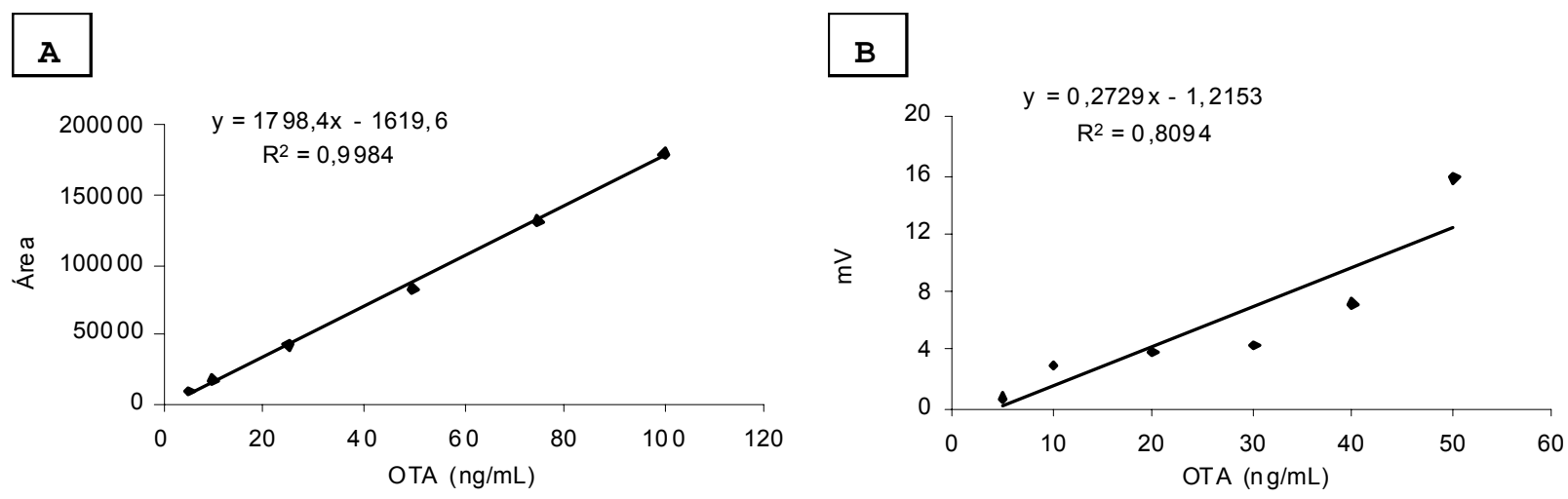

Figura 1. Curva padrão de OTA obtida por CLAE (A) e espectrofluorimetria (B). O eixo X representa a concentração de OTA (ng/mL); o eixo Y representa a área (CLAE) e o potencial de fluorescência (espectrofluorimetria).

Entre estes prováveis fatores, aponta-se o efeito físico de microplaca de poliestireno utilizada para proceder à leitura no espectrofluorímetro. A microplaca de 96 poços empregada no ensaio consistia de poços com forma de "U", sendo a leitura em cada poço realizada com a radiação eletromagnética de excitação formando um ângulo em "V" com a de emissão. Sendo assim, qualquer alteração no posicionamento da microplaca interferia no ângulo de incidência/emissão das radiações e conseqüentemente, na potência de fluorescência.
Neste contexto, a utilização de microplaca causar baixa linearidade da curva padrão.

A Tabela 1 compara a análise de OTA por CLAE e espectrofluorimetria em amostras de café artificialmente contaminados (10-50 ng/g). Os teores de OTA obtidos por CLAE variaram de 9,21 - 41,20 $\mathrm{ng} / \mathrm{g}$, em relação a $3,50-18,36 \mathrm{ng} / \mathrm{g}$ por espectrofluorimetria (Tabela 1), obtendo-se relação média espectrofluorimetria/CLAE de 0,45 e coeficiente de correlação (r) de 0,79 (Figura 2).

Tabela 1. Análise de OTA por CLAE e espectrofluorimetria em grãos de café artificialmente contaminados.

\begin{tabular}{|c|c|c|c|c|c|c|}
\hline \multirow[t]{2}{*}{ Amostras } & \multirow{2}{*}{$\begin{array}{c}\text { OTA } \\
\text { adicionada } \\
(\mathrm{ng} / \mathrm{g})\end{array}$} & \multicolumn{2}{|c|}{ OTA recuperada (ng/g) } & \multicolumn{2}{|c|}{ Espectrofluorimetria/CLAE } & \multirow[t]{2}{*}{$\mathrm{r}^{\mathrm{b}}$} \\
\hline & & CLAE & Espectrofluorimetria & Relação & $\mathrm{X}^{\mathrm{a}}$ & \\
\hline 1 & 10 & 9,21 & 3,50 & 0,38 & & \\
\hline 2 & 10 & 9,54 & 5,89 & 0,62 & & \\
\hline 3 & 20 & 18,49 & 9,69 & 0,52 & 0,45 & 0,79 \\
\hline 4 & 20 & 17,65 & 8,36 & 0,47 & & \\
\hline 5 & 50 & 38,53 & 18,36 & 0,48 & & \\
\hline 6 & 50 & 41,20 & 10,00 & 0,24 & & \\
\hline
\end{tabular}

a(x) média da relação espectrofluorimetria/CLAE.

${ }^{b}(\mathrm{r})$ coeficiente de correlação espectrofluorimetria/CLAE. 


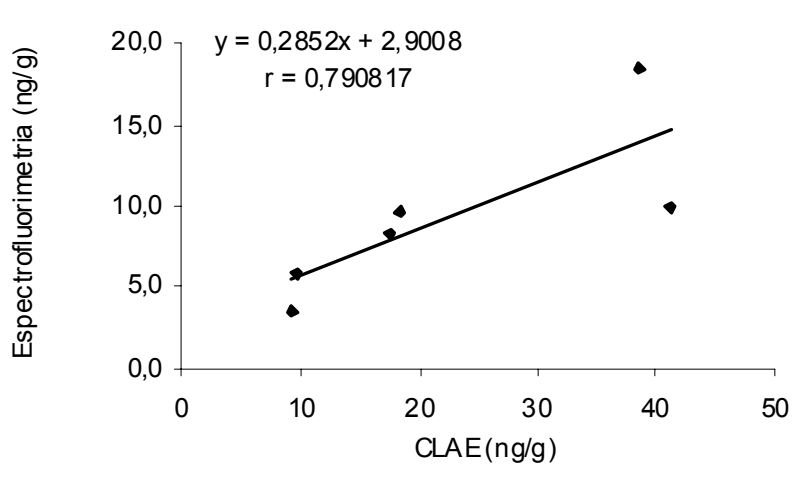

Figura 2. Correlação entre CLAE e espectrofluorimetria na determinação de OTA em grãos de café. A equação de regressão linear $\mathrm{y}=0,2852 \mathrm{x}+2,9008$ resultou em coeficiente de correlação de 0,79 . O valor X representa a concentração de OTA (ng/g) determinada por CLAE, enquanto que $\mathrm{Y}$ representa os dados da mesma amostra determinada por espectrofluorimetria.

Considerando que a quantificação de OTA por ambas as metodologias seja baseada na deteç̧ão de fluorescência emitida pela toxina, esperava-se correlação adequada entre CLAE e espectrofluorimetria. Todavia, os baixos valores de relação (espectrofluorimetria/CLAE $=0,45$ ) e coeficiente de correlação $(0,79)$ seriam justificados pela presença de componentes alimentares não removidos na etapa de limpeza por CCD, com provável interferência na análise espectrofluorimétrica, dificultando a absorção e emissão de radiação eletromagnética pela OTA. O fato diminuiu a fluorescência emitida, subestimando a concentração de OTA na amostra. Na CLAE, a eluição através de coluna cromatográfica separou os componentes da matriz alimentar, evitando a interferência na quantificação de OTA, permitindo obter resultados mais confiáveis.

A Tabela 2 mostra a eficiência de CLAE e espectrofluorimetria na recuperação de OTA em grãos de café. As amostras artificialmente contaminadas com 10, 20 e $50 \mathrm{ng} / \mathrm{g}$ de OTA apresentaram recuperação de 79,73 a 93,75 \% (média de $87,94 \%$ ) por CLAE, enquanto que os valores foram de 28,39 a 46,93 \% (média de 40,14\%) por espectrofluorimetria. Estes resultados demonstraram baixa confiabilidade e eficiência inadequada da espectrofluorimetria na análise de OTA em grãos de café, devendo-se prosseguir com o estudo inserindo modificação técnica, seguida de ensaios de padronização, visando certificação da metodologia para ensaio de rotina.

Silva et al. (2005), utilizando CCD e espectrofluorimetria acoplada a coluna de imunoafinidade, obtiveram correlação de 97 \% na análise de aflatoxinas em 40 amostras de milho. $\mathrm{Na}$ espectrofluorimetria, os teores da micotoxina variaram de 0,68 a 58,66 $\mu \mathrm{g} / \mathrm{g}$, enquanto que em CCD variaram de 3 a $54 \mu \mathrm{g} / \mathrm{g}$.

Tabela 2. Recuperação de OTA por CLAE e espectrofluorimetria em grãos de café artificialmente contaminados.

\begin{tabular}{cccccc}
\hline \multirow{2}{*}{ Metodologia } & \multicolumn{2}{c}{ OTA (ng/g) } & \% Recuperação & CV (\%) & $\begin{array}{c}\text { Média } \\
\text { Recuperação }\end{array}$ \\
\cline { 2 - 3 } & adicionada & recuperada & & & \\
\hline \multirow{2}{*}{ CLAE $^{\mathrm{a}}$} & 10 & $9,38 \pm 0,20$ & $93,75 \pm 1,99$ & 2,12 & \\
& 20 & $18,07 \pm 0,51$ & $90,35 \pm 2,57$ & 2,85 & $87,94 \pm 6,68$ \\
& 50 & $39,86 \pm 1,59$ & $79,73 \pm 3,17$ & 3,98 & \\
Espectrofluorimetria $^{\mathrm{b}}$ & 10 & $4,69 \pm 1,34$ & $46,93 \pm 13,36$ & 28,46 & \\
& 20 & $9,02 \pm 0,76$ & $45,11 \pm 3,79$ & 8,39 & $40,14 \pm 12,45$ \\
& 50 & $14,20 \pm 4,56$ & $28,39 \pm 9,13$ & 32,15 & \\
\hline
\end{tabular}

${ }^{a}$ média \pm desvio padrão de duas repetições em duplicata.

${ }^{\mathrm{b}}$ média \pm desvio padrão de duas repetições em duplicata. 
Chiavaro et al. (2002) obtiveram correlação de 0,98 entre CLAE e fluorimetria na análise de OTA em presunto, utilizando coluna de imunoafinidade na limpeza de amostras. A recuperação de OTA por fluorimetria foi de $82,9 \% \mathrm{em}$ amostras artificialmente contaminadas com 0,7 a $9,7 \mu \mathrm{g} / \mathrm{Kg}$, evidenciando a eficácia da técnica. Apesar de resultados confirmarem a eficiência da coluna de imunoafinidade acoplada a espectrofluorimetria, o seu custo ainda dificulta o amplo uso na rotina laboratorial.

Embora a análise espectrofluorimétrica de OTA em café não tenha atingido resultado plenamente satisfatório, o ensaio preliminar mostrou perspectivas otimistas para prosseguir com estudo inserindo novas condições e ensaios de padronização. Em adição, a simplicidade, sensibilidade, baixo custo, uso reduzido de solventes tóxicos e a ausência de contato do aparelho com solventes apontam a espectrofluorimetria como alternativa promissora para triagem de micotoxinas em alimentos.

\section{Agradecimentos}

À CAPES/MEC (Coordenadoria de Aperfeiçoamento de Pessoa de Nível Superior) pela bolsa de Mestrado. Ao CNPq (Conselho Nacional de Pesquisa e Desenvolvimento Científico e Tecnológico) pelo apoio financeiro. Ao IAPAR-PR pelo fornecimento das amostras de café.

\section{Referências}

CHIAVARO, E.; LEPIANI, A.; COLLA, F.; BETTONI, P.; PARI, E.; SPOTTI, E. Ochratoxin A determination in ham by immunoaffinity clean-up and a quick fluorometric method. Food Additives and Contaminants, Parma-Italy, v. 19, n. 6, p. 575-581, 2002.

CHU, F. S. Immunoassays for analysis of mycotoxins. Journal of Food Protection, Des Moines, v. 47, n. 7, p. 562-569, 1984.

ESKOLA, M.; KOKKONEN, M.; RIZZO, A. Application of manual and automated systems for purification of ochratoxin A and zearalenone in cereals with immunoaffinity columns. Journal of Agricultural and Food Chemistry, Easton, v. 50, n. 1, p. 41-47, 2002.

FOOD AND AGRICULTURE ORGANIZATION OF UNITED NATIONS - FAO. Reducing ochratoxin $A$ in coffee. Disponível em: <http://www.coffee-ota.org/ ota_what.asp $>$. Acesso em: 20 mar. 2006.

FINK-GREMMELS, J.; JAHN, A.; BLOM, M. J. Toxicity and metabolism of ochratoxin A. Natural Toxins, New York, v. 3, n. 4, p. 214-220, 1995.

FURLANI, R. P. Z.; OLIVEIRA, P. L. C.; SOARES, L. M. V. Avaliação de métodos para determinação de ocratoxina A em cafés verdes e torrados. Revista do Instituto Adolfo Lutz, São Paulo, v. 58, n. 2, p. 87-98, 1999.

GROSSO, F.; SAÏD, S.; MABROUK, I.; FREMY, J. M.; CASTEGNARO, M.; JEMMALI, M.; DRAGACCI, S. New data on the occurrence of ochratoxin A in human sera from patients affected or not by renal diseases in Tunisia. Food and Chemical Toxicology, Oxford, v. 41, n. 8, p. 1133-1140, 2003.

JODBAUER, J.; MAIER, N. M.; LINDNER, W. Towards ochratoxin A selective moleculary imprinted polymers for solid-phase extraction. Journal of Chromatography A, Amsterdam, v. 945, n. 1, p.45-63, 2002.

PETRACCO, M. Melhoramento da qualidade do café pela redução do crescimento de fungos. In: ENCONTRO NACIONAL DE MICOTOXINAS, 9., 1998, Florianópolis. Anais... Florianópolis: UFSC, 1998. p. 1-20.

PITTET, A.; TORNARE, D.; HUGGETT, A.; VIANI, R. Liquid chromatographic determination of ochratoxin $\mathrm{A}$ in pure and adulterated soluble coffee using an immunoaffinity column cleanup procedure. Journal of Agricultural and Food Chemistry, Washington, v. 44, n. 11,p. 3564-3569, 1996.

PITTET A.; ROYER D. Rapid, low cost thin layer chromatographic screening method for the detection of ochratoxin A in green coffee at control level of $10 \mathrm{mg} / \mathrm{kg}$. Journal of Agricultural and Food Chemistry, v. 50, n.2, p.243-247, 2002.

SILVA, M.; BERND, L. P.; SILVA, A. P.; MARCO, H. E.; FRACAO, F. A.; CAMPANER, J. A.; MARONEZE, D. M.; ROSMANINHO, J. F.; UCHOA, P. P. M.; ITANO, E. N.; ONO, M. A.; KAWAMURA, O.; ONO, E. Y.; HIROOKA, E. Y. Comparação entre metodologia analítica química e imunoquímica na quantificação de aflatoxinas em milho. In: ENCONTRO NACIONAL DE ANALISTAS DE ALIMENTOS, 14., 2005, Goiânia. Resumos... Goiânia: Sociedade Brasileira de Analistas de Alimentos-Regional, 2005.p. 137. 
SOARES, L. V. Noções de controle de qualidade e segurança de qualidade analítica. In: Curso básico de instrumentação para analistas de alimentos e fármacos. Barueri: Manole, 2006. p. 43-51.
SYDENHAM, E. W.; SHEPHARD, G. S. Chromatographic and allied methods of analysis for selected mycotoxins. In: GILBERT, J. Progress in Food Contaminants Analysis. London: Blackie Academic \& Professional, 1996. p.65-146.

TOLEDO, L. Os diferentes sabores e preços do café brasileiro. Revista Exportar e Gerência, Brasília, n. 20, p. $18-24,2000$. 
